\title{
Genetic factors associated with recurrent pregnancy loss
}

\begin{abstract}
The aetiology behind recurrent pregnancy loss is unknown in about $50 \%$ of cases despite having a lot of known factors. Two to five percent of RPL occur due to a genetic cause. A structural or a numerical abnormality in the chromosomes of the parents or in the foetus can result in pregnancy loss. Several genes like those that are involved in angiogenesis, oxidative stress, clotting and inflammation have also been associated with RPL. Recent advances such as the use of microarrays and next generation sequencing for detecting the genetic abnormalities in RPL patients, in the aborted foetuses and in the embryos in the form of preimplantation genetic screening can help to better diagnose and treat RPL patients.
\end{abstract}

Volume 7 Issue 6 - 2017

\author{
Bibhas Kar, Linda Catherine B \\ Center for Genetic Studies and Research,The Madras Medical \\ Mission, India \\ Correspondence: Bibhas Kar, Center for Genetic Studies and \\ Research, The Madras Medical Mission, J.J. Nagar east, Chennai \\ 600037,Tel 044- 26568000,Email drbibhas_kar@yahoo.co.in
}

Received: May 29, 2017 | Published: September 18, 2017

\section{Introduction}

Recurrent pregnancy loss (RPL) also referred to as recurrent miscarriage or recurrent spontaneous abortion has been a cause for bad obstetric history along with still births, intrauterine fetal deaths and intrauterine growth retardation (IUGR). According to the Practice Committee of the American Society for Reproductive Medicine, for epidemiological studies, three or more pregnancy losses are considered as RPL whereas, for clinical purposes, two pregnancy losses in the first trimester constitute RPL. ${ }^{1}$

Though there are several known causes that lead to RPL such as uterine structure abnormalities, infection from TORCH agents, chromosomal abnormalities, endocrine factors and environmental factors, $50 \%$ of the causes are not known., ${ }^{2,3}$ Genetic causes contribute $2-5 \%$ of the aetiology for RPL and they may be due to chromosomal abnormalities or gene mutations. ${ }^{3}$ This paper has been subdivided into two in order to discuss the cytogenetic and the molecular defects observed in association with RPL. Genetic studies play an essential role in diagnosing RPL and helps in better understanding of the condition.

\section{Cytogenetic Defects}

About $50 \%$ of the aborted foetuses have chromosomal abnormalities as a result of nondisjunction and thus cytogenetic evaluation becomes very crucial in diagnosing spontaneous abortions. In a study involving 151 recurrent abortion patients with a first trimester abortion, $7.3 \%$ of the mothers had chromosomal abnormalities of which X chromosome mosaicism dominated followed by reciprocal translocations and Robertsonian translocations. $2.1 \%$ of the fathers had chromosomal defects with $\mathrm{X}$ chromosome mosaicism and inversions. Trisomy, polyploidy and monosomy dominated among the aborted fetuses. ${ }^{4,5}$ Thus, structural and numerical defects in the chromosome of both the parents and the foetus can lead to abortion.

\section{Aneuploidy}

An addition or deletion of chromosomes in the foetus or in the parents can lead to an abortion. Numerical chromosomal abnormalities in parents, resulting in abortion are not as common as structural abnormalities but of them, aneuploidies involving the $\mathrm{X}$ chromosome are the most commonly observed. ${ }^{6}$ In a study conducted in China, only $0.18 \%$ of the 137 RPL carriers had chromosomal abnormalities whereas balanced translocations dominated. ${ }^{7}$ A comparative study was done between spontaneous abortion (SA) and RPL using the aborted foetuses and it was found that the number of chromosomal abnormalities were significantly higher in RPL than in SA. The abnormalities observed in aborted foetus of RPL patients were trisomies, monosomy X, polyploidy and other structural aberrations. Among the trisomies, trisomy 16 and 22 were the most commonly observed and in SA cases, in addition to trisomy 16 and 22, trisomy 13, 18 and monosomy $\mathrm{X}$ were also prominent. ${ }^{8}$ When the sperm samples from men suffering from RPL were analysed, chromosome 16 disomy was very common and contributed to $60 \%$ of the cases. ${ }^{9}$ Sperm aneuploidy test for chromosomes $18, \mathrm{X}$ and $\mathrm{Y}$ was conducted which revealed that $10.6 \%$ of sperms had a chromosomal aneuploidy in RPL patients whereas in the control group, there was an abnormality of only $1.5 \%$. Moreover, an increase in the rate of sex chromosome disomy was also observed. ${ }^{10}$

\section{Structural chromosomal abnormalities}

Structural defects were observed in $92.9 \%$ of the parental population and all of them were balanced translocations. Balanced translocation is the most common type of chromosomal aberration in an RPL patient and counselling the parents helps in getting a better pregnancy outcome. The chance for their offspring to have an unbalanced translocation can be minimized. ${ }^{6,11,12}$ When chromosomal abnormality carriers and non-carriers were compared, no significant difference was observed between them in the number of live births. This study confirms that apart from chromosomal defects, there are other aetiologies to RPL.

The involvement of more than 2 breakpoints in 3 chromosomes gives rise to a complex nature of rearrangement and a balanced complex chromosomal rearrangement involving chromosomes 3,18 and 21 with 4 break points was observed in a family by using highresolution banding and three colour FISH. ${ }^{13}$ A retrospective study conducted on 1415 couples showed that only $0.1 \%$ of the couples had complex chromosomal rearrangements. ${ }^{14}$ Thus, though uncommon, complex chromosomal rearrangements also contribute to RPL.

The role of chromosomal variants such as inv(9)(p11q12), $\mathrm{qh}+$ heteromorphisms in chromosomes 1, 9,16 and $\mathrm{Y}$; pseudo satellites in chromosomes 13,14, 15, 21 and 22 and fragile sites in chromosome 16 
and 17 in recurrent pregnancy loss has been a topic of debate for long of which, inv(9), 1qh+, 9qh+, fra(17) were of statistical significance in the Romanian population. ${ }^{15}$ In the Indian population, inv(9) and $1 \mathrm{qh}+$ were commonly observed. ${ }^{16}$

An aberration in the heterochromatin regions of the long and short arms of the $\mathrm{Y}$ chromosome have been observed in infertile men.
Therefore, Yqh+ and Yqh- may be associated with an increased risk of abortion. ${ }^{17}$ But, microdeletions in the azoospermia region of the $\mathrm{Y}$ chromosome (AZFa, AZFb and AZFc regions) were not found to be associated with RPL. ${ }^{18}$ Numerical and structural chromosomal abnormalities observed in the parents and the abortus are listed in Table $1 \& 2$.

Table I Structural aberrations observed in RPL patients and in the aborted foetus s,6,7,17 $^{4}$

\begin{tabular}{|c|c|c|}
\hline & Females & Males \\
\hline \multirow{27}{*}{$\begin{array}{l}\text { Structural } \\
\text { Abnormalities }\end{array}$} & \multicolumn{2}{|l|}{$46, X X, t(2 ; 12)$} \\
\hline & $46, X X, t(2 ; 4)(p 24 ; q \mid 3)$ & $46, X Y, t(I ; 2)(p 22 ; q 3 I)$ \\
\hline & $46, X X, t(3 ; 8)(q 25 ; p 21), 9 q h+$ & $46, X Y, t(I ; I I)(p 22 ; q 23)$ \\
\hline & $46, X X, t(3 ; 6)(p 25 ; q \mid 3)$ & $45, X Y, t(I ; I I)(q 25 ; q 23), \operatorname{rob}(I 5 ; 22)(q I 0 ; q I 0)$ \\
\hline & $46, X X, t(6 ; 9)(p 21 ; p 23)$ & $46, X Y, t(I ; I I)(q 42 . I ; q \mid 3.4)$ \\
\hline & $46, X X, t(6 ; I I)(q 2 I ; q 23.3)$ & $46, X Y, t(2 ; 3)(p \mid 3 ; q 27)$ \\
\hline & $46, X X, t(8 ; 14)(p 21 ; q 32)$ & $46, X Y, t(3 ; 20)(p|4.1 ; p| 3)$ \\
\hline & $46, X X, t(8 ; 15)(q 22 ; q 15)$ & $46, \mathrm{XY}, \mathrm{t}(4 ; 17)(\mathrm{q} 2 \mathrm{I} ; \mathrm{pl} 3)$ \\
\hline & $46, X X, t(9 ; 16)(q 22.1 ; p I I . I)$ & $46, X Y, t(4 ; 20)(q 22 ; p \mid I .2)$ \\
\hline & $46, X X, t(9 ; 20)(q 2 I ; p I I .2)$ & $46, X Y, t(4 ; 22)(q 34 ; q I)$ \\
\hline & $46, X X, t(10 ; 16)(q 25.1 ; p \mid 2)$ & $46, \mathrm{XY}, \mathrm{t}(8 ; \mathrm{I} 8)(\mathrm{q} 22.3 ; \mathrm{q} 2 \mathrm{I.I})$ \\
\hline & $46, X X, t(I I ; \mid 7)$ & $46, X Y, t(8 ; 22)(p 21 ; q \mid 3 . I)$ \\
\hline & $46, X X, t(I I ; \mid 8)(q 25 ; 92 I)$ & $46, X Y, t(|3 ;| 8)(q 21 ; q 22)$ \\
\hline & $46, X X, t(13 ; 16)(q 34, q 12)$ & $45, X Y, \operatorname{rob}(\mid 4 ; 2 I)(q|0 ; q| 0)$ \\
\hline & $45, X X, \operatorname{rob}(13 ; \mid 3)(q|0 ; q| 0)$ & $45, X Y, \operatorname{rob}(|3 ;| 4)(q|0 ; q| 0), 22$ pstk+ \\
\hline & $45, X X, \operatorname{rob}(\mid 4 ; 2 I)(q 10 q \mid 0)$ & $46, X Y, \operatorname{inv}(7)(p \mid 5 q 22)$ \\
\hline & $46, X X, \operatorname{rob}(|5 ; 2|)(q|0 ; q| 0)$ & 45,XY,inv(9)(pl|q|3),rob(I4; 22)(q|0;q|0) \\
\hline & $45, X X, \operatorname{rob}(15 ; 22)(q 10 ; q 10)$ & $46, X Y, \operatorname{inv}(18) q 10$ \\
\hline & $45, X X, \operatorname{rob}(21 ; 22)(q|0 ; q| 0)$ & $46, X Y, I$ qh+ \\
\hline & $46, X X, \operatorname{inv}(I)(p|| q \mid 2)$ & $46, X Y, 9 q h+$ \\
\hline & $46, X X$,inv (9p) & $46, X Y q h+, 9 q h+$ \\
\hline & $46, X X$, I qh+ & $46, \mathrm{XYqh}+$ \\
\hline & $46, X X, 9 q h+$ & $46, \mathrm{XYqh}-$ \\
\hline & $46, X X, 16 q h+$ & $46, X Y, 15 p s+$ \\
\hline & $46, X X, 13 p s t k+p s+$ & $46, X Y, 2$ Ips+ \\
\hline & $46, X X, 22 p s t k+$ & $46, X Y, 2$ I cenh+ \\
\hline & $46, X X, 2$ Ipstk+ & \\
\hline & $\begin{array}{l}46, X X / 47, X X X / 45, X \\
46, X X / 47, X X X\end{array}$ & \\
\hline Numerical & $46, X X / 49, X X X X X / 45, X$ & \\
\hline Abnormalities & $46, X X / 49, X X X X X$ & 46,XY/47,XXY \\
\hline & $45, X / 46, X X$ & \\
\hline & $47, X X X$ & \\
\hline
\end{tabular}

Table 2 Chromosomal aberrations observed in the aborted tissue ${ }^{6}$

\begin{tabular}{|c|c|}
\hline Structural abnormalities & Numerical abnormalities \\
\hline $\begin{array}{l}\text { 45,XX, rob(22;22)(q I0;q I0) } \\
\text { 46,XX,der(7)t(3;7)(p2I.3;q35) } \\
\text { 46,XY,der(I4)t(8; I4)(p2I;q32)mat } \\
\text { 46,XY,r(I3;। } 4),+13\end{array}$ & $\begin{array}{l}47, X Y,+5 \\
47, X Y,+8 \\
47, X Y,+12 \\
\text { Trisomy I } \\
47, X Y,+14 \\
47, X Y,+15 \\
47, X Y,+16 \\
\text { Trisomy I8 } \\
48, X X Y,+18 \\
47, X Y,+20 \\
\text { Trisomy } 21 \\
47, X Y,+22 \\
48, X X,+14,+21 \\
45, X \\
45, X / 46, X X \\
46, X Y / 46, X X \\
69, X X Y \\
69, X X X \\
69, X X Y, i n v(9)(p|| q \mid 3)\end{array}$ \\
\hline
\end{tabular}




\section{Gene mutations}

Mutations in the genes that are involved in maintaining pregnancy can result in an abortion. Oxidative stress, thrombophilic factors and immunologic factors such as Human Leucocyte antigen and cytokine gene mutations are contributing factors to RPL. Polymorphisms in the oestrogen receptor (ER), progesterone receptor (PR) and androgen receptor (AR) have been studied in association with RPL. Skewed X chromosome inactivation was also found to be significantly associated with RPL. ${ }^{19}$

\section{Oxidative stress}

Common anti-oxidant levels were lower in RPL patients when compared to normal ones in whole blood and in the plasma. Subsequently, the level of known oxidants was significantly higher in RPL patients than in women with successful a pregnancy. ${ }^{20} \mathrm{~A}$ study of oxidative stress related genes was conducted and ABCB1 (rs1045642-T), COMT (rs4680-A), GPX4 (rs713041-T), and OGG1 (rs1052133-G) alleles were associated with RPL. ${ }^{21}$ A Tet-mev 1 transgenic mouse model with a defective mitochondrial respiratory chain causing an increase in oxidative stress was studied. On observation, it was found that the pregnant mice showed placental angiodysplasia leading to a hypoxic condition. This further lead to the inflammation of the placenta and abnormal angiogenesis and resulted in spontaneous abortion. Thus oxidative stress influences the occurrence of RPL.22

\section{Thrombophilic factors}

Coagulation disorders are a risk factor for thrombotic events and can lead to pregnancy loss. A number of thrombophilic mutations have been studied in association with RPL. Angiotensin I- converting enzyme $(A C E)$ gene plays a part in the renin-angiotensin system (RAS) which regulates blood pressure and thereby helps in maintaining pregnancy. A study involving 149 patients with $\geq 3$ abortions and a meta-analysis that was performed with 1192 patients showed no association between the insertion/deletion polymorphism in intron 16 of $A C E$ gene \& RPL. ${ }^{23}$ Though $A C E$ gene showed no significance for the I/D polymorphism in Iranian population, a strong association was seen between Plasminogen activator inhibitor 1 (PAI-1) 4G/5G polymorphism and RPL. PAI-1 4G polymorphism was seen in $17 \%$ of the cases and in 5\% of the controls. Both $A C E$ and PAI-1 genes control and regulate the coagulation of blood. While PAI-1 inhibits fibrinolytic reactions, $A C E$ converts angiotensin I to angiotensin II which helps in the synthesis of PAI- $1 .{ }^{24}$ Evaluation of coagulation factors such as anti-thrombin III, protein $\mathrm{C}$, protein $\mathrm{S}$, factor V Leiden and lupus anticoagulant in the plasma of spontaneous abortion patients revealed that at least one abnormality was seen in $42.7 \%$ of cases with the most common being protein $\mathrm{C}$ deficiency followed by abnormal AT III and lupus anticoagulant. ${ }^{25}$ Factor II prothrombin (PTm) G20210A mutation plays an important role in RPL. ${ }^{17}$ Coagulation factor XIII and $\beta$ fibrinogen are both important for the coagulation of blood and a meta-analysis that was performed revealed that only FXIII Val34Leu polymorphism had an association with RPL. ${ }^{26}$

MTHFRC677Tpolymorphismhasbeenshowntobesignificantamong other thrombophilia genes. ${ }^{6}$ MTHFR (10-methylentetrahydrofolate reductase) is an enzyme involved in folate metabolism and polymorphisms in the gene coding for this enzyme revealed no significant difference between the cases and the controls. However, the combination of rs1801133 and/or rs1801131 alleles were more common in patients with spontaneous abortion. ${ }^{27}$ In another study, MTHFR polymorphisms $677 \mathrm{C} / \mathrm{T}$ and $1298 \mathrm{~A} / \mathrm{C}$ and methionine synthase reductase (MTRR) polymorphism $66 \mathrm{~A} / \mathrm{G}$ were studied and $677 \mathrm{~T} / \mathrm{T}$ of $M T H F R$ and $66 \mathrm{~A} / \mathrm{G}$ polymorphism of $M T R R$ were higher in the patients. ${ }^{28}$ A polymorphism in these genes affects methionine production and in turn the pregnancy outcome by influencing DNA synthesis and repair.

\section{Angiogenesis genes}

Nitric oxide (NO) mediates the relaxation of vascular smooth muscles. Low production of NO can affect the supply of oxygen and nutrition to the foetus. VNTR polymorphism in intron 4 and the Glu298Asp polymorphism in exon 7 are the most common polymorphisms in endothelial nitric oxide synthase (eNOS). A tumor suppressor gene $p 53$ mediates inflammation, transcriptional regulation and controls the growth of the foetus by playing a role in angiogenesis. Less or over production of p53 as a result of mutation can lead to the death of the foetus. Vascular endothelial growth factor (VEGF) plays a crucial role in the angiogenic development of the foetus and the placenta. The common polymorphism in $V E G F$ that has been associated with RPL is $(-1154 G / A)$ in the promoter region. ${ }^{29}$

\section{Immunologic factors}

Human Leucocytic Antigen: Specific DP, DQ and DR alleles of Class II MHC have an increased risk of miscarriage and a $14 \mathrm{bp}$ polymorphism in the UTR region of $H L A-G$ has been found in RPL patients. HLA-G is a minor Class I molecule that is present on the cytotrophoblast. It mediates pregnancy by controlling the NK cells and by suppressing the proliferation of the T lymphocytes. A decrease in the amount of $H L A-G$ has been observed in women with RPL. Allelic variants of $H L A-G$ such as 0105 N, 0106, 010401, 010108 have been associated with lower level of sHLA-G and contributes to RPL susceptibility. ${ }^{30}$ WHO Nomenclature Committee for factors of the HLA System has accepted around $15 H L A-G$ alleles in the coding region. ${ }^{31}$

Cytokines: Cytokines are signalling molecules that help to communicate between the trophoblast and the decidual cells. Th1 dominant immunity was thought to be associated with miscarriage but a Th2 dominance has also been associated with RSA. Thus the Th1- Th2 paradigm has been expanded to Th1/Th2/Th17and Treg cell paradigm. Th1 cells produce pro-inflammatory cytokines like TNF $\alpha$, TNF $\beta$, IFN $\gamma$, IL1, IL2 which activate the cytotoxic T lymphocytes thereby initiating a cell mediated response. IL 4, IL 5, IL 6, IL 10 and IL 12 are anti-inflammatory cytokines that are produced by Th2 cells and they result in the production of antibodies by the B cells ${ }^{32} \mathrm{~A}$ number of cytokine polymorphisms and their role in RPL have been studied as cytokines play a very important part in pregnancy. Several studies have shown an association but the results are inconsistent and vary with populations. The levels of IL-18, leukemia inhibitory factor (LIF), and migration inhibitory factor (MIF) in blood and IL12 , IFN- $\gamma$, intracellular adhesion molecule-1 (ICAM-1) in tissue have been found to be significantly different between RPL patients and fertile patients. ${ }^{33}$

In Iran, polymorphisms in the promoter region and in the introns of FOXP3 (forkhead or winged helix transcription factor) gene, a gene involved in the development of Treg cells were analysed of which $-924 \mathrm{~A} / \mathrm{G}$ and $-20 \mathrm{G} / \mathrm{A}$ were statistically different between the cases and the controls. Reduction in the expression of FOXP3 results in a decrease in Treg production and this can affect the balance of the cytokines that are produced. ${ }^{34}$

Complement activation: The complement system which consists of serum proteins is important in maintaining maternal tolerance against 
the foetus during pregnancy. This system consists of three pathways, the classical, alternative and the lectin pathway based on the factor that initiates the complement system. Cryy in mouse is a homolog of Membrane co-factor protein (MCP) otherwise called CD 46 and decay accelerating factor (DAF), and a deficiency of this protein leads to embryonic death as a result of placental inflammation due to excess C3 deposition. ${ }^{35,36}$ The levels of complement split proteins in the blood and the expression of complement inhibitory proteins at the foeto-maternal surface were analysed and it was found that the level of C5a was higher in spontaneous abortion patients over those who underwent an an elective abortion. Furthermore, the regulatory proteins CD46 and CD55 were lower in patients with spontaneous abortion. ${ }^{37}$ The higher expression of the regulatory proteins may be due to mutations that occur in the genes coding for CD46 and C4 binding protein (C4BP) in RPL patients . ${ }^{36}$

\section{Microarrays and whole exome sequencing}

Chromosomal microarray has been considered to be a good technique to detect abnormalities in the product of conception over karyotyping as it has a higher success rate and it provides more information. It can be performed even on dead cells however maternal contamination has to be ruled out. ${ }^{38}$ Whole genome and whole exome sequencing (WES) can potentially be used to detect novel mutations in RPL patients. A study was conducted on four families suffering from RPL and it was noted that of the 4 families, 2 showed a mutation in DYNC2H1 (Dynein Cytoplasmic 2 Heavy Chain 1) and $A L O X 15$ (arachidonate 15-lipoxygenase) genes. Both these genes are involved in the early development of the foetus. Though WES has been found to be efficient, the study used a very small sample size. ${ }^{39}$ Hence WES performed with a bigger sample size can shed light into the deleterious genes related to RPL.

\section{Preimplantation Genetic Diagnosis (PGD)/ Preimplantation Genetic Screening (PGS)}

In the case of genetic abnormality, the patients are advised for genetic counselling which will help them to understand the risks involved in future pregnancies. A genetic study of their family for a similar aberration can be done to get a better picture will help in decision making. ${ }^{40}$ For translocation carriers with 3 or more pregnancy losses, it was seen that with PGD, the live birth rate was $87 \%$. Thus PGD can help RPL patients with better pregnancy outcomes and also reduce the time taken to conceive. ${ }^{41}$ However, in a review that was conducted, it was seen that on comparing live birth rates between patients who conceived naturally and those who conceived after PGD, the results were similar. ${ }^{42}$ PGS using Next Generation Sequencing (NGS) to screen the entire genomic content of an embryo prior to implantation coupled with advances in artificial reproductive techniques can be effective in reducing pregnancy loss in RPL patients. ${ }^{43}$

\section{Conclusion}

Chromosomal studies show that the abnormalities vary between parents and the aborted foetuses. While balanced translocations are more common in the RPL parents, trisomies dominate among the aborted samples. Thus testing of both the parents and the foetus proves to be important for future pregnancies. Several genes have been studied in association with RPL, the most important being the genes involved in the coagulation of blood. Screening for common chromosomal defects and gene mutations needs to be implemented along with other routine tests for the diagnosis of RPL.

\section{Conflicts of Interest}

The authors declare no conflict of interest regarding the publication of this article.

\section{Acknowledgements}

The authors are thankful to the Management Committee of The Madras Medical Mission for providing research infrastructure and facilities.

\section{References}

1. Evaluation and Treatment of Recurrent Pregnancy Loss: A Committee Opinion. Fertil Steri. 2012;198(5): 1103-1111.

2. Rai R, Regan L Recurrent miscarriage. The Lancet. 2006; 368(9535):601-611.

3. Ford HB, Schust DJ Recurrent Pregnancy Loss: Aetiology, Diagnosis, and Therapy. Rev Obstet Gynecol. 2009; 2(2):76-83.

4. Gonçalves RO, Santos WV, Sarno M, et al. Chromosomal abnormalities in couples with recurrent first trimester abortions. Rev Bras Ginecol Obstet. 2014;36(3):113-117.

5. Alonso López AG1, Bermejo Huerta S, Hernández Galván R, et al. Cytogenetic diagnosis of first trimester spontaneous abortion. Ginecol Obstet Mex. 2011; 79(12): 779-784.

6. Ocak Z, Özlü T, Ozyurt O Association of recurrent pregnancy loss with chromosomal abnormalities and hereditary thrombophilias. Afr Health Sc. 2013; i 13(2):447-452.

7. Dong Y, Li LL, Wang RX, Yu XW, Yun X, et al. Reproductive outcomes in recurrent pregnancy loss associated with a parental carrier of chromosome abnormalities or polymorphisms. Genet Mol Res. 2014; 13(2):2849-2856.

8. Choi TY, Lee HM, Park WK, Jeong SY, Moon HS Spontaneous abortion and recurrent miscarriage: A comparison of cytogenetic diagnosis in 250 cases. Obstet Gynecol Sci. 2014;57(6):518-525.

9. Neusser M, Rogenhofer N, Dürl S, Ochsenkühn R, Trottmann M, et al. Increased chromosome 16 disomy rates in human spermatozoa and recurrent spontaneous abortions. Fertil Steril. 2015;104(5):1130-1137.

10. Zidi-Jrah I, Hajlaoui A, Mougou-Zerelli S, Kammoun M, Meniaoui I, et al. Relationship between sperm aneuploidy, sperm DNA integrity, chromatin packaging, traditional semen parameters, and recurrent pregnancy loss. Fertil Steril. 2016;105(1): 58-64.

11. Mogib El-Dahtory F. Chromosomal abnormalities as a cause of recurrent abortions in Egypt. Indian J Hum Genet. 2011;17(2):82-84.

12. Gaboon NE, Mohamed AR, Elsayed SM, et al. Structural chromosomal abnormalities in couples with recurrent abortion in Egypt. Turk J Med Sci. 2015;45(1):208-213.

13. Liao Y, Wang L, Zhang D, et al. Identification of a balanced complex chromosomal rearrangement involving chromosomes 3, 18 and 21 with recurrent abortion: case report. Mol Cytogenet. 2014;7(1):39.

14. Nonaka T, Ooki I, Enomoto $\mathrm{T}$, et al. Complex chromosomal rearrangements in couples affected by recurrent spontaneous abortion. Int J Gynaecol Obstet. 2015;128(1):36-39.

15. Mierla D, Stoian V. Chromosomal Polymorphisms Involved in Reproductive Failure in the Romanian Population. Balkan J Med Genet. 2012;15(2):23-28.

16. Berkovitz A, Eltes F, Ellenbogen A, et al. Does the presence of nuclear vacuoles in human sperm selected for ICSI affect pregnancy outcome? Hum Reprod. 2006;21(7):1787-1790. 
17. Karatas A, Eroz R, Albayrak M, et al. Evaluation of Chromosomal Abnormalities and Common Trombophilic Mutations in Cases with Recurrent Miscarriage. Afr Health Sci. 2014;14(1):216-222.

18. Pereza N, Črnjar K, Buretić-Tomljanović A, et al. Y chromosome azoospermia factor region microdeletions are not associated with idiopathic recurrent spontaneous abortion in a Slovenian population: association study and literature review. Fertil Steri. 2013;199(6):16631667.

19. Su M, Lin S, Chen Y. Association of sex hormone receptor gene polymorphisms with recurrent pregnancy loss: a systematic review and meta-analysis. Fertil Steri. 2011;196(6):1435-1444.

20. Ghneim HK, Alshebly MM. Biochemical Markers of Oxidative Stress in Saudi Women with Recurrent Miscarriage. J Korean Med Sci. 2016;31(1):98-105.

21. Khadzhieva MB, Lutcenko NN, Volodin IV, et al. Association of oxidative stress-related genes with idiopathic recurrent miscarriage. Free Radic Res. 201448(5):534-541.

22. Ishii T, Miyazawa M, Takanashi Y, et al. Genetically induced oxidative stress in mice causes thrombocytosis, splenomegaly and placental angiodysplasia that leads to recurrent abortion. Redox Bio. 2014;12:679 685 .

23. Pereza N, Ostojić S, Zdravčević M, et al. Insertion/deletion polymorphism in intron 16 of ACE gene in idiopathic recurrent spontaneous abortion case-control study, systematic review and meta-analysis. Reprod Biomed Online. 2016;32(2):237-246.

24. Shakarami F, Akbari MT, Zare Karizi S. Association of plasminogen activator inhibitor-1 and angiotensin converting enzyme polymorphisms with recurrent pregnancy loss in Iranian women. Iran J Reprod Med. 2015;13(10):627-632.

25. Besharat M, Tabandeh A, Keshtkar A, et al. Evaluation of Some Plasma Coagulation Factors in Women with Spontaneous Miscarriage. Int $J$ Fertil Steril. 2016;9(3):309-312.

26. Li J, Wu H, Chen Y, et al. Genetic association between FXIII and $\beta$ fibrinogen genes and women with recurrent spontaneous abortion: a meta- analysis. J Assist Reprod Genet. 2015;32(5):817-825.

27. Hubacek JA, Rynekrova J, Kasparova D, et al. Association of MTHFR genetic variants C677T and A1298C on predisposition to spontaneous abortion in Slavonic population. Clin Chim Acta. 2015;440:104-107.

28. Zhu L. Polymorphisms in the methylene tetrahydrofolate reductase and methionine synthase reductase genes and their correlation with unexplained recurrent spontaneous abortion susceptibility. Genet Mol Res. 2015;14(3):8500-8508.

29. Su M, Lin S, Chen Y. Genetic association studies of angiogenesis- and vasoconstriction-related genes in women with recurrent pregnancy loss: a systematic review and meta-analysis. Hum Reprod Update. 2011;17(6):803-812.
30. Arjmand F, Ghasemi N, Mirghanizadeh S, et al. The balance of the immune system between HLA-G and NK cells in unexplained recurrent spontaneous abortion and polymorphisms analysis. Immunol Res 64(3): 785-790.

31. Hviid T (2006) HLA-G in human reproduction: aspects of genetics, function and pregnancy complications. Hum Reprod Update. 2016;12(3):209-232.

32. Saini V, Arora S, Yadav A, et al. Cytokines in recurrent pregnancy loss. Clin Chim Acta. 2011;412(9-10):702-708.

33. Comba C, Bastu E, Dural O, et al. Role of inflammatory mediators in patients with recurrent pregnancy loss. Fertil Steril. 2015;104(6):1467e1-1474.e1.

34. Naderi-Mahabadi F, Zarei S, Fatemi R, et al. Association study of forkhead box P3 gene polymorphisms with unexplained recurrent spontaneous abortion. J Reprod Immunol. 2015;110:48-53.

35. Mao D, Wu X, Deppong C, Friend L, et al. Negligible Role of Antibodies and C5 in Pregnancy Loss Associated Exclusively with C3-Dependent Mechanisms through Complement Alternative Pathway. Immunity. 200319(6):813-822.

36. Liszewski MK, Atkinson JP. Complement regulator CD46: genetic variants and disease associations. Hum Genomics. 2015;9(1):7.

37. Banadakoppa M, Chauhan M, Havemann D, et al. Spontaneous abortion is associated with elevated systemic C5a and reduced mRNA of complement inhibitory proteins in placenta. Clin Exp Immunol. 2014;177(3):743-749.

38. Page JM, Silver RM. Genetic Causes of Recurrent Pregnancy Loss. Clin Obstet Gynecol. 2016;59(3):498-508.

39. Qiao Y, Wen J, Tang F, et al. Whole exome sequencing in recurrent early pregnancy loss. Mol Hum Reprod. 2016;22(5):364-372.

40. Chetty M, Duncan W. Investigation and management of recurrent miscarriage," Obstetrics, Gynaecology \& Reproductive Medicine. $2015 ; 25(2): 31-36$

41. Fischer J, Colls P, Escudero T, et al. Preimplantation genetic diagnosis (PGD) improves pregnancy outcome for translocation carriers with a history of recurrent losses. Fertil Steril. 2010;94(1):283-289.

42. Franssen M, Musters A, van der Veen F, et al. Reproductive outcome after PGD in couples with recurrent miscarriage carrying a structural chromosome abnormality: a systematic review. Hum Reprod Update. 201117(4):467-475

43. Martín J, Cervero A, Mir P, et al. The impact of next-generation sequencing technology on preimplantation genetic diagnosis and screening. Fertil Steril. 2013;99(41054-1061. 